\title{
L'enseignement de la réanimation au sein du DESC de médecine d'urgence en France : état des lieux et perspectives
}

\author{
Teaching Intensive Care Medicine as Part of the DESC Specialist Emergency Medicine \\ Qualification in France: Current Situation and Outlook
}

\author{
K. Tazarourte \\ Reçu le 30 juillet 2015 ; accepté le 25 août 2015 \\ (C) SRLF et Lavoisier SAS 2015
}

La médecine d'urgence (MU) est devenue en quelques décennies un acteur majeur des systèmes de soins dans le monde. Les médecins urgentistes sont souvent le maillon initial de la prise en charge des patients en situation de détresse vitale. Une des spécificités de la MU est de pouvoir (devoir) prendre en charge, à la phase initiale, l'ensemble des pathologies aiguës relevant de spécialités d'organe et de réaliser, si nécessaire, des techniques de réanimation au profit de ces patients. L'utilisation de ces techniques (intubation trachéale, ventilation mécanique, évacuation des plèvres, abords veineux, médicaments spécifiques, monitorage, ultrasons...), en préhospitalier ou dans un service d'urgence, doit être régie par les mêmes impératifs de qualité, de sécurité et de conformité aux bonnes pratiques que dans un service de réanimation. L'habileté dans la réalisation de gestes techniques est essentielle, mais ne résume pas la qualité d'une prise en charge. L'impact pronostique (vital et fonctionnel) dépend également des qualités et du savoir-faire intellectuel, relationnel et organisationnel du praticien en charge du patient [1]. Une bonne compréhension des mécanismes physiopathologiques, des options thérapeutiques, de l'évolution de l'état du patient, qu'il faudra savoir anticiper, mais aussi la capacité à délivrer une information claire et précise aux familles sont des impératifs à maîtriser dès la phase préhospitalière $[2,3]$. Les enjeux de l'enseignement de la réanimation, adapté pour les médecins urgentistes, sont bien plus larges que le simple apprentissage des gestes.

L'enseignement de la MU est régi, en France depuis 2004, par un DESC de type 1 non qualifiant. C'était une avancée majeure qui permettait de faire évoluer une capacité de $\mathrm{MU}$, sans obligation de stage en réanimation, en un enseignement universitaire structuré sur deux années et imposant un

\footnotetext{
K. Tazarourte $(\bowtie)$

Service des urgences et du centre de médecine hyperbare, pôle urgence-Samu-anesthésie-réanimation, 5, place d'Arsonval, CHU Édouard-Herriot, F-69003 Lyon, France

e-mail : Karim.tazarourte@chu-lyon.fr
}

apprentissage clinique et théorique de six mois en service de réanimation médicale et/ou chirurgicale. Toutefois, l'enseignement actuel de la réanimation au sein du DESC est jugé insuffisant par les jeunes médecins urgentistes. Lors d'une enquête nationale prospective déclarative réalisée en 2014 auprès des DESC de MU en deuxième année (208 DESC inclus sur 436 contactés), plus de la moitié des jeunes urgentistes s'estimaient insuffisamment formés pour les gestes techniques de réanimation et pour la prise en charge des urgences vitales [4]. L'intubation trachéale et la gestion d'un accès difficile aux voies aériennes, la pose d'un drain thoracique ou d'un cathéter intraosseux et la pratique de l'échographie étaient considérées par plus de $60 \%$ d'entre eux comme insuffisamment enseignées [4]. Paradoxalement, la pose de voies veineuses centrales ou celle d'un cathéter artériel posaient moins de problèmes $(70 \%$ de satisfaction pour la formation). Les urgentistes du DESC, tous issus du DES de médecine générale, exprimaient comme besoin prioritaire une formation accentuée en anesthésie locorégionale et en réanimation polyvalente, par le biais de semestres complémentaires et de formations techniques et relationnelles spécifiques par simulation haute et basse fidélité [4]. Il est manifeste que la maquette du DES de médecine générale déroule une formation qui n'est probablement pas le meilleur prérequis pour la MU. Cette étude confirme l'impérieuse nécessité de repenser le cursus universitaire de formation à la $\mathrm{MU}$ en France. En Europe, 15 pays (Belgique, Finlande, Hongrie, Irlande, Islande, Italie, Luxembourg, Malte, Pologne, République tchèque, Roumanie, Royaume-Uni, Suède, Slovénie, Slovaquie) ont placé la MU au rang de spécialité à part entière, avec un cursus de formation spécifique, dont la durée varie de quatre à cinq ans [5]. Depuis 2011, l'Union européenne des médecins spécialistes (UEMS), sous l'impulsion de l'European Society of Emergency Medicine (EUSEM), a créé la section de MU, dont le but est de promouvoir, dans tous les pays membres de l'Union européenne, la MU en tant que spécialité, de proposer une 
harmonisation des formations et des cursus professionnels et d'initier des procédures de certification à l'instar du Competency-Based Training in Intensive Care Medicine in Europe (CoBaTrICE) [2,5].

En France, le principe de la création d'un DES de MU a été acté par les tutelles, et un référentiel métier a été élaboré conjointement [6]. Ce référentiel métier insiste particulièrement sur les compétences à acquérir, cognitives (savoir), techniques (savoir-faire), comportementales (savoir être, savoir travailler en équipe) réflexives (réfléchir sur sa pratique et réinvestir les résultats de cette réflexion dans l'action). Le cursus sera de quatre années, avec au minimum deux semestres en service de réanimation polyvalente et/ou de soins intensifs. L'UEMS préconise un cursus de cinq ans, mais la durée totale du cursus est probablement moins importante que la capacité à certifier les compétences obligatoires à acquérir avant d'exercer. Aux États-Unis, où la MU est une spécialité à part entière avec un cursus de quatre ans, la moitié des 219 urgentistes interrogés en fin de formation estimaient être très peu à l'aise pour instaurer une ventilation mécanique et régler les paramètres du respirateur [7]. Un volume horaire peu important $(<3$ heures dans l'année) et une faible pratique étaient les principales causes énoncées. Dans cette étude, les urgentistes estimaient que leurs stages en réanimation n'étaient pas suffisamment encadrés par des seniors. Un des biais est qu'aux États-Unis le réglage du respirateur est délégué à une catégorie professionnelle inconnue chez nous, les respiratory therapists [7]. Toutefois, les urgentistes les plus à l'aise pour gérer correctement une ventilation mécanique étaient ceux qui avaient eu plus de cinq heures de formation théorique et une pratique régulière. L'intégration des urgentistes en stage de réanimation doit se faire à un moment de leur cursus où ils ont une maturité technique et cognitive suffisante pour pouvoir être pleinement intégrés dans l'équipe et correctement encadrés.

Si la certification des compétences en fin de formation initiale est essentielle et plutôt bien codifiée, à défaut d'être homogène, le maintien des compétences (recertification) est un enjeu crucial et encore insuffisamment abordé en France. La pratique de gestes avancés de réanimation (intubation trachéale, ventilation mécanique ou non invasive, arrêt cardiaque, drainage thoracique...) en MU concerne un nombre variable de patients, selon que le médecin urgentiste exerce en centre hospitalier à haut ou bas volume d'activité. L'intubation trachéale en service mobile d'urgence et de réanimation (SMUR) va concerner un patient sur dix, et la gestion d'un traumatisé grave devient plus rare, alors que le niveau de technicité doit être élevé [8]. Les courbes de désapprentissage ont rarement été étudiées en MU, et pourtant, il s'agit là d'un enjeu. À l'avenir, un niveau de pratique clinique minimale, technique ou situationnelle, doit être exigé pour garantir le maintien des compétences de l'urgentiste. La recertification des médecins est bien codifiée dans le monde anglo-saxon [9]. Elle doit trouver sa place en France, en particulier par le biais de la simulation haute-fidélité.

En conclusion, l'enseignement de la réanimation en MU, théorique et pratique, va être considérablement renforcé par l'arrivée du DES de MU. Les liens entre urgentistes et réanimateurs sont ceux de partenaires évidemment complémentaires, qui travaillent de concert dans une chaîne de survie des patients les plus graves. Sécuriser le maillon initial de la chaîne (les urgentistes) est le garant d'un meilleur pronostic. Le maintien des compétences, au regard d'un niveau d'activité minimal, par le biais d'une recertification est essentiel. L'Europe est un puissant vecteur d'amélioration. L'EUSEM, en proposant des cursus de formation harmonisés, nous tire vers le haut. À n'en pas douter, les passerelles entre urgentistes et réanimateurs vont s'élargir. Vivement le DES de MU ! en 2016 ?

Liens d'intérêts : L'auteur déclare ne pas avoir de lien d'intérêt.

\section{Références}

1. Wessman BT, Fuller BM (2014) Training emergency physicians to meet the critical care needs in the United States: a consensus of two. Crit Care Med 42:e677-e8

2. Bion J, Rothen HU (2014) Models for intensive care training. A European perspective. Am J Respir Crit Care Med 189:256-62

3. Debaty G, Ageron FX, Minquet L (2015) More than half the families of mobile intensive care unit patients experience inadequate communication with physicians. Intensive Care Med 41:1291-8

4. Mesnier T, Mimoz O, Oriot D, Ghazali DA (2015) DESC de médecine d'urgence : comment les jeunes urgentistes apprennentils leur spécialité ? Première enquête nationale. Ann Fr Med Urg 5:95-102

5. Totten V, Bellou A (2013) Development of emergency medicine in Europe. Acad Emerg Med 20:514-21

6. Nemitz B, Carli P, Carpentier F, et al (2012) Référentiel métier compétences pour la spécialité de médecine d'urgence. Ann Fr Med Urg 2:125-38

7. Wilcox SR, Seigel TA, Strout TD (2015) Emergency medicine residents' knowledge of mechanical ventilation. J Emerg Med 48:481-91

8. Tazarourte K, Cesaréo E, Sapir D, et al (2013) Update in prehospital management of major trauma. Ann Fr Anesth Reanim 32:477-82

9. Smith-Coggins R, Marco CA, Baren JM, et al (2015) American Board of Emergency Medicine Report on Residency Training Information (2014-2015). Ann Emerg Med 65:584-94 